\title{
Correction: The absence that makes the difference: choroidal abnormalities in Legius syndrome
}

\author{
Arianna Tucci $^{1}$ - Veronica Saletti ${ }^{2}$ - Francesca Menni ${ }^{1}$ - Claudia Cesaretti ${ }^{3}$ - Giulietta Scuvera ${ }^{1} \cdot$ Silvia Esposito $^{2}$. \\ Giulia Melloni ${ }^{3} \cdot$ Susanna Esposito $^{1,5} \cdot$ Donatella Milani $^{1} \cdot$ Cristina Cereda $^{4} \cdot$ Mario Cigada $^{6} \cdot$ Laura Tresoldi $^{6}$.

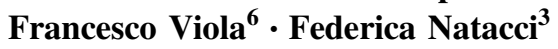

Received: 19 September 2017 / Accepted: 19 September 2017

(c) The Japan Society of Human Genetics 2018

Correction to: Journal of Human Genetics advance online publication 27 July 2017; https://doi.org/10.1038/jhg.2017.78

Since the publication of the above article, the authors of the above paper have noticed an error in two of the Contributing Author's affiliation. "4Genomic and Post-genomic Center IRCCS 'C. Mondino', National Neurological Institute, Pavia, Italy" should have been "Pediatric Clinic, Università degli Studi di Perugia, Perugia, Italy" and "5ediatric Clinic, Università degli Studi di Perugia, Perugia, Italy" should have been "Genomic and Postgenomic Center IRCCS 'C. Mondino', National Neurological Institute, Pavia, Italy"

The authors would like to apologize for this mistake.
Federica Natacci

f.natacci@policlinico.mi.it

1 Department of Pathophysiology and Transplantation, Pediatric Highly Intensive Care Unit, Università degli Studi di Milano, Fondazione IRCCS Ca' Granda Ospedale Maggiore Policlinico, Milan, Italy

2 Developmental Neurology IRCCS Foundation, 'C. Besta' Neurological Institute Milan, Milan, Italy

3 Medical Genetics Unit Woman, Child and Newborn department,
IRCSS Foundation, Ca' Granda-Ospedale Maggiore Policlinico, Milan, Italy

4 Genomic and Post-genomic Center IRCCS 'C. Mondino', National Neurological Institute, Pavia, Italy

5 Pediatric Clinic, Università degli Studi di Perugia, Perugia, Italy

6 Department of Clinical Sciences and Community Health, Ophthalmological Unit, Ca' Granda Foundation-Ospedale Maggiore Policlinico, Università degli Studi di Milano, Milan, Italy 